\title{
Required Emergency Room or Doctor Visit
}

National Cancer Institute

\section{Source}

National Cancer Institute. Required Emergency Room or Doctor Visit. NCI Thesaurus.

Code C102436.

An event in which a visit to the emergency room or to the doctor is considered essential to prevent permanent impairment or damage. 